\title{
Pulmonary Rehabilitation in Patients Recovering from COVID-19
}

\author{
Elisabetta Zampogna ${ }^{a}$ Mara Paneroni $^{b}$ Stefano Bellic ${ }^{c}$ Maria Aliani ${ }^{d}$ \\ Alessandra Gandolfo ${ }^{e}$ Dina Visca ${ }^{a}{ }^{f}$ Maria Teresa Bellanti ${ }^{c}$ \\ Nicolino Ambrosino ${ }^{g}$ Michele Vitacca ${ }^{b}$
}

aRespiratory Rehabilitation of the Institute of Tradate, Istituti Clinici Scientifici Maugeri, IRCCS, Tradate, Italy;

${ }^{b}$ Respiratory Rehabilitation of the Institute of Lumezzane, Istituti Clinici Scientifici Maugeri, IRCCS, Brescia, Italy;

'Respiratory Rehabilitation of the Institute of Veruno, Istituti Clinici Scientifici Maugeri, IRCCS, Veruno, Italy;

${ }^{\mathrm{d}}$ Respiratory Rehabilitation of the Institute of Bari, Istituti Clinici Scientifici Maugeri, IRCCS, Bari, Italy; ${ }^{\text {Respiratory }}$

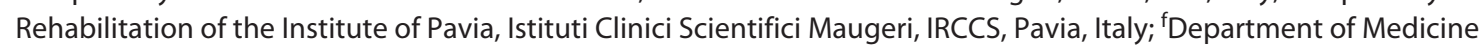
and Surgery, Respiratory Diseases, University of Insubria, Varese-Como, Italy; ${ }^{9}$ Respiratory Rehabilitation of the Institute of Montescano, Istituti Clinici Scientifici Maugeri, IRCCS, Montescano, Italy

\section{Keywords}

Exercise training · Exercise capacity · Dyspnoea · Pulmonary rehabilitation

\section{Abstract \\ Background: In hospitalized patients recovering from the SARS-coronavirus-2 disease 19 (COVID-19), high prevalence of muscle weakness and physical performance impairment has been observed. Objectives: The aim of this study was to evaluate the effectiveness of pulmonary rehabilitation in these subjects in a real-life setting. Methods: Retrospective data analysis of patients recovering from COVID-19, includ- ing those requiring assisted ventilation or oxygen therapy, consecutively admitted to an in-patient pulmonary rehabili- tation program between April 1 and August 15, 2020. Short Physical Performance Battery (SPPB: primary outcome), Bar- thel Index (BI), and six-min walking distance were assessed as outcome measures. Results: Data of 140 patients were analyzed. After rehabilitation, patients showed improve- ments in SPPB \{from: (median [IQR]) 0.5 (0-7) to 7 (4-10), $p<$ $0.001\}$ and $\mathrm{BI}$ (from 55 [30-90] to 95 [65-100], $p<0.001$ ), as well as in other assessed outcome measures. The proportion}

of patients unable at admission to stand, rise from a chair and walk was significantly reduced $(p<0.00)$. Conclusions: Pulmonary rehabilitation is possible and effective in patients recovering from COVID-19. Our findings may be useful to guide clinicians taking care of patients surviving COVID-19 infection.

(c) 2021 S. Karger AG, Basel

\section{Introduction}

The SARS-coronavirus-2 disease 19 (COVID-19) pandemic has caused dramatic effects throughout the world, with tens of millions of people infected and $>1$ million casualties [1]. Approximately $80 \%$ of patients have mild to moderate, $15 \%$ severe disease, and $5 \%$ have critical illness [2]. The disease can cause major alveolar damage resulting in hypoxemic acute respiratory failure requiring mechanical ventilation in a high proportion of cases [3, 4]. The long-term physical, psychological, and cognitive impairment of both survivors and their caregivers remain to be described [5]. In hospitalized patients without any prior motor limitation, recovering from COVID-19, a 
high prevalence of muscle weakness and physical performance impairment has been observed [6]. Furthermore in patients requiring intensive care unit stay, the muscle impairment could be related, among others, to systemic inflammation, mechanical ventilation, sedation, and prolonged bed rest [7]. In addition, many authorities have forbidden for long-time travels and people moving, resulting also in prevention of attendance to in- or outpatient pulmonary rehabilitation programs.

Despite clinical indications and modalities of pulmonary rehabilitation have been proposed by international guidelines and recommendations [8-10], the tolerance to and the effects of such programs in patients recovering from COVID-19 remain to be elucidated. However, we cannot wait for well-designed randomized controlled trials to be published before starting these interventions in daily clinical practice, as the number of COVID-19 patients increases rapidly every day. Therefore, the aim of this multicenter retrospective study was to report the effectiveness of pulmonary rehabilitation in subjects recovering from COVID-19 in a real-life setting.

\section{Methods}

The study was approved by the Istituti Clinici Scientifici (ICS) Maugeri Ethics Committee (CEC 2279; March 12, 2020). At admission to ICS institutions, patients gave their informed consent for the scientific use of their data. As a retrospective analysis, the study was not registered.

\section{Patients}

This study was conducted on the Automated Integrated Health Care Record database of patients recovering from COVID-19 with negative RT-PCR test for SARS-CoV-2, consecutively admitted for inpatient pulmonary rehabilitation between April 1 and August 15,2020 , to ICS Maugeri hospitals in Italy (Bari, Lumezzane, Tradate, Pavia, and Veruno) and referral institutions for pulmonary rehabilitation, diagnosis, and care for post-acute and chronic subjects $[11,12]$. Patients were transferred from intensive and subintensive care units, pneumology units, or general wards where they had been managed, including for COVID-19 induced acute respiratory failure requiring or not either invasive or noninvasive ventilation (NIV). Patients were admitted to selected areas and received drug therapy according to the evolving information and current research [13]. One or more of the following drugs - chloroquine, steroids, and anticoagulants - had been prescribed in addition to therapy for patients' underlying comorbidities. Exclusion criteria were persistent positive RT-PCR test and reported prior clinical conditions preventing active mobilization.

\section{Measurements}

At admission the following data were collected: demographics, anthropometrics, number, and diagnosis of comorbidities by the Cumulative Illness Rating Scale (CIRS), including the Cumulative
Illness Rating Score Comorbidities Index (CI) and the Cumulative Illness Rating Score Severity Index (SI) [14]. CIRS-CI was calculated assigning to each item a score between 0 (none) and 4 (extremely severe), total score reflecting the mean value of the first 13 items. CIRS-CI was obtained by the sum of the items with score $\geq 3$. Length of stay (LoS) in referring hospitals, use of mechanical ventilation, either invasive or NIV, and arterial blood gases were recorded as well.

With the safety procedures and wearing appropriate personal protective equipment [15], the following outcome measures were assessed when allowed by patients' clinical conditions and safety or organizational issues.

- Motor performance was assessed by the Barthel Index (BI) [16]. The total BI score ranges from 0 (maximum level of dependency) to 100 (complete autonomy). A score $\leq 70$ corresponds to severe dependency.

- The lower extremity function was assessed by means of the Short Physical Performance Battery (SPPB) $[17,18]$ with the predicted normal values of Bergland et al. [19]. The SPPB total score results from the sum of 3 components: standing balance, 4-m walking test (4MWT) and standing from sitting position 5 times (5-STS). The total SPPB score ranges from 0 to 12 : $1-2$ : severe; 3-8 moderate disability; 9-12 normal. One point is considered as the minimal clinically important difference (MCID) for SPPB [20]. i.For tests of standing balance included side-byside, semi-tandem, and tandem standing, the subjects were timed until they moved or $10 \mathrm{~s}$ had elapsed. The subjects were given a score of 0 if bedridden, 1 if they could hold only the side-by-side standing position, a score of 2 if they could hold a semi-tandem position but were unable to hold a tandem position for $>2 \mathrm{~s}$, a score of 3 if they could stand in the tandem position for 3-9 s, and a score of 4 if they could stand in the tandem position for $10 \mathrm{~s}$. ii. The $4 \mathrm{MWT}$ was performed twice. The faster time spent to complete the test was used for scoring as follows: unable to walk a score: $0 ;>8.7 \mathrm{~s}$ : $1 ; 8.7-6.2 \mathrm{~s}$ : $2 ; 6.2-4.8$ s: $3 ;<4.8$ s: 4 [21]. iii. Quartiles of time required to complete the 5-STS were used for scoring as follows: unable to rise: $0, \geq 16.7$ s: $1 ; 13.7-16.6$ s: $2 ; 11.2-13.6$ s: 3 ; and $\leq 11.1$ s: 4 [22].

- Exercise tolerance was assessed by the 6-m walking test (6MWT) [23] using the predicted values of Enright et al. [24]. The baseline value of patients unable to perform the test was considered as 0 for analysis.

\section{Pulmonary Rehabilitation}

Health-care operators experienced in pulmonary rehabilitation were trained to manage patients with COVID-19 wearing appropriate personal protective equipment [15]. A multidisciplinary program according to the Italian Position Paper was applied in all centers involved [9]. Type, intensity, timing and modality of intervention were tailored to the individual patient according to age, clinical severity, length of immobilization, comorbidities, starting from a minimum of one, 20-min daily session up to two-three, 30min daily sessions.

According to the SPPB total score, patients were allocated either to individual (level A if SPPB $<6$ with a physiotherapist/patient ratio $1: 1$ ) or group (level B if $S P P B \geq 6$ with a physiotherapist/ patient ratio 1:4-5) sessions. The level A program might include or be limited to one or more of the following: mobilization, active exercises and free walking, peripheral limb muscle activities, shoulder, and full arm circling. The level B program might include 


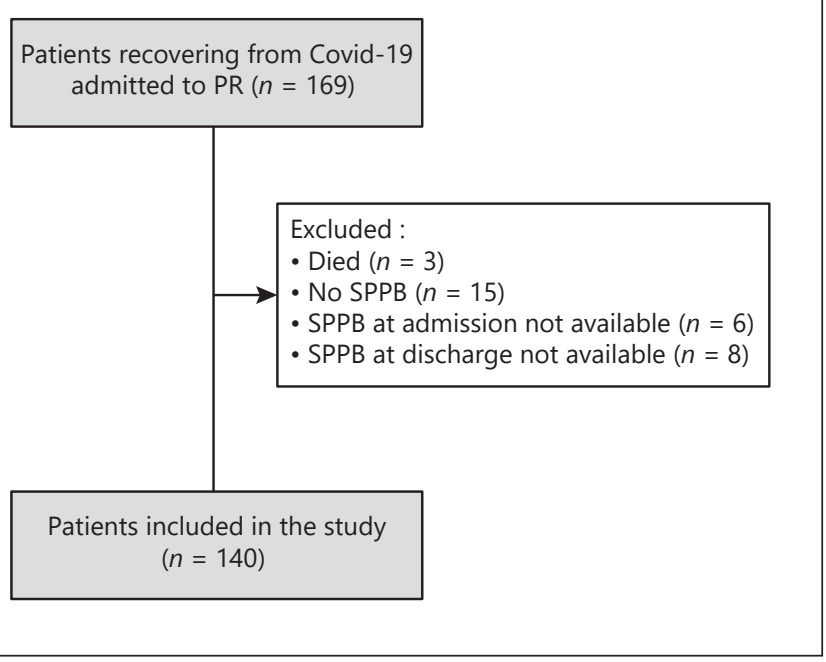

Fig. 1. CONSORT diagram of patient selection. SPBB, Short Physical Performance Battery; BI, Barthel Index.

or be limited to one or more of the following: callisthenic, strengthening, balance exercise, and paced walking. All exercises could be performed without devices or using gymnastic tools such as balls, canes, balance boards, or light weights bands [9].

Patients in level B with higher physical autonomy were also trained on cycle-ergometer at low-intensity exercises ( $<3.0 \mathrm{MET}$ ). The initial training workload was chosen starting from 0 and progressively increasing until patients scored their dyspnea and/or leg fatigue as 4 or 5 on a modified 10-point Borg Scale [25]. Thereafter the progression of intensity was according to Maltais et al. [26]: the workload was increased by $5 \mathrm{~W}$ when patients scored less or equal to 3 , was unchanged when the Borg score was 4 or 5 , and was reduced by $5 \mathrm{~W}$ for scores of $>5$. Also, chest physiotherapy such as bronchial hygiene techniques by using disposable devices with self-management in order to avoid the risk of environmental contamination $[15,27]$, and lung expansion procedures were performed when required.

Subjects were re-assessed on a daily base in order to adjust the type, intensity, timing and modality of the intervention. According to patient's individual conditions, the program might include also nutritional and psychological assessment.

\section{Statistical Analysis}

Statistical analysis was performed using STATA 11 (StataCorp LLC). Data for continuous variables were expressed as median (interquartile range; IQR) and binary outcomes were described as percentage (\%). SPPB total score and 6MWT were also defined as mean \pm standard deviation (SD). The change in SPPB total score was the primary outcome. The pre-to-post outcome measure changes were evaluated by Wilcoxon signed rank test or by $\chi^{2}$ tests. Odd ratio analysis was performed to evaluate the risk of improvement in SPPB above the MCID (1 point) [22] according to the baseline characteristics. $p<0.05$ was considered as statistically significant.
Table 1. Demographic, anthropometric, physiological, and clinical characteristics of patients in study
Age, years

Male, $n(\%)$

BMI, $\mathrm{kg} / \mathrm{m}^{2}$

LoS in acute hospitals, days

Previous NIV, $n$ (\%)

Previous oxygen need, $n(\%)$

$\mathrm{PaO}_{2} / \mathrm{FiO}_{2}(n=130)$

$\mathrm{PaO}_{2}, \mathrm{~mm} \mathrm{Hg}(n=130)$

$\mathrm{PaCO}_{2}, \mathrm{~mm} \mathrm{Hg}(n=130)$

$\mathrm{pH}(n=130)$

CIRS-SI, score

CIRS-CI, score
Previous invasive ventilation, $n(\%)$
$71.0(61.5-78.0)$

$95(67.8)$

$25.2(23.2-29.3)$

$47.0(33.5-64.0)$

$56(40.0)$

$70(50.0)$

$117(83.6)$

$338.1(310.5-371.4)$

$72.4(67.1-84.0)$

$37.8(34.00-42.1)$

$7.43(7.40-7.45)$

$1.6(1.60-2.1)$

$4.0(3.0-5.0)$
Data are expressed as $n(\%)$ or median (IQR). BMI, body mass index; LoS, length of stay; NIV, noninvasive ventilation; $\mathrm{PaO} 2$, arterial oxygen tension; $\mathrm{PaCO} 2$, arterial carbon dioxide tension; $\mathrm{FiO} 2$, inspired oxygen fraction; CIRS-SI, Cumulative Illness Rating Score Severity Index; CIRS-CI, Cumulative Illness Rating Score Comorbidities Index; IQR, interquartile range.

\section{Results}

The study flow chart is shown in Figure 1. Out of 169 patients admitted, 140 (Bari: 5, Lumezzane: 51, Pavia: 5, Tradate: 56, Veruno: 23), with assessments of SPPB both before and after the program, were included. Demographics, anthropometrics, physiological, and clinical characteristics of patients are shown in Table 1. Sixteen 1 $\%$ and $4.4 \%$ of patients suffered from chronic obstructive pulmonary disease and asthma, respectively.

Patients had suffered from long LoS in acute care hospitals, and a high proportion of patients had undergone mechanical ventilation either invasive (including some tracheostomized) or NIV. At admission, $6.2 \%$ of patients still had a tracheostomy, $7.1 \%$ were still under NIV, and $23.8 \%$ still used oxygen supplementation (mean fraction of inspired oxygen $\left.\left[\mathrm{FiO}_{2}\right]=0.23 \pm 0.05\right)$. LoS in our institutions was $24.0(19.0-34.0)$ days.

Patient performed 60 (38-84) sessions corresponding to $2.8(1.0-3.8)$ daily sessions. Thirty-one percent of patients performed only level A interventions, $7 \%$ only level B, and $62 \%$ shifted from level A to level B. About $70 \%$ of patients completed their program into level B. None of patients had to step down from level B to A. Eighty-five patients (61.0\%) underwent $14.0(8.0-19.0)$ sessions of cycle-ergometer endurance training with an initial workload of $5.0(1.2-10.0)$ up to $30.0(20.0-40.0)$ W. Additional physiotherapy other than endurance training was 


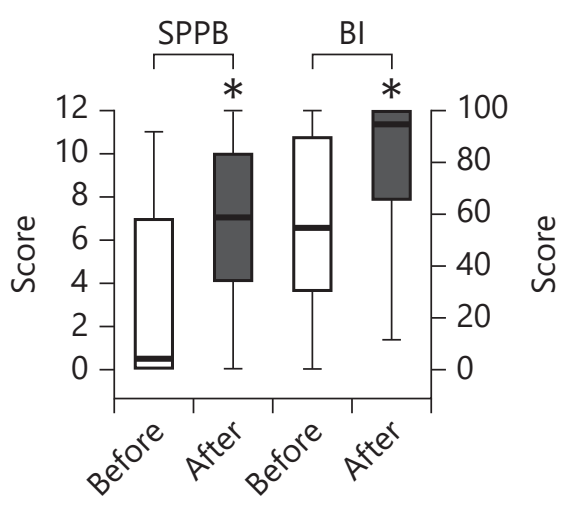
rehabilitation. SPBB, Short Physical Performance Battery; BI, Barthel Index.
Fig. 2. Changes in SPPB and BI scores before and after pulmonary

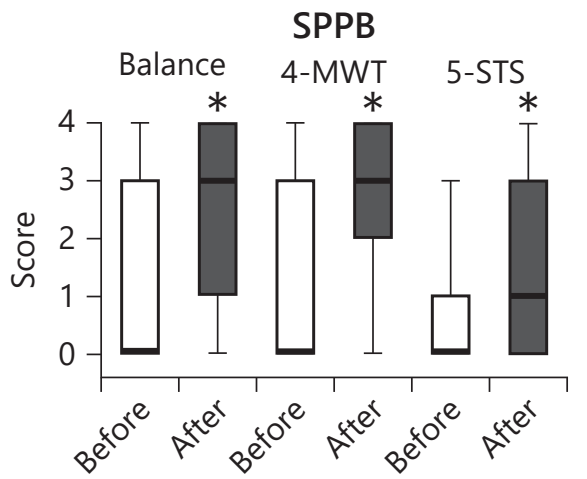

Fig. 3. Change of SPPB components (balance, $4 \mathrm{MWT}$, and 5-STS) before and after rehabilitation. The lines indicating the medians of before rehabilitation data correspond to zero. SPBB, Short Physical Performance Battery; BI, Barthel Index; 4MWT, 4-m walking test; 5-STS, sitting position 5 times.

was significantly reduced after rehabilitation $(p<0.00)$. The odd ratio analysis showed that an improvement above 1 point in SPPB total score was significantly associated with the median baseline SPPB total score $<3$ (odds ratio [OR] 3.5 [SE 1.3], $p=0.00$ ), previous use of NIV (OR 3.0 [SE 1.1] $p=0.002)$ and baseline inability to perform 6MWT (OR 2.3 [SE 0.9], $p=0.001$ ).

The BI improved after the program from 55.0 (30.0 $90.0)$ to $95.0(65.0-100.0)(p=0.00)$. Forty-two (30.0\%) subjects were able to perform the $6 \mathrm{MWT}$ at admission as compared to $81(57.8 \%)$ post treatment. The distance walked during the $6 \mathrm{MWT}$ by these 81 patients was 285.0 (232.0-370.0; mean \pm SD $298.2 \pm 116.7 \mathrm{~m})$. Data for $6 \mathrm{MWT}$ improvement in 42 patients able also at admission to perform the test are shown on Table 2.

\section{(4.1-9.0) and 16.7 (12.0-22.9) seconds, respectively.}

Change in the SPPB total score after the program was 3.0 (1.0-6.0), and 89 patients (63.6\%) reached the MCID. Final SPPB score was $7(4-10)(6.9 \pm 3.8)$. The SPPB, \% predicted improved from $4.2(0.0-58.3)$ to 66.7 (37.8107.1) $(p=0.00)$. After the program, the number of patients reporting score $\leq 2$ was reduced to $23(16.4 \%)$. Figure 3 shows the SPPB components (balance, 4MWT, and 5-STS) before and after rehabilitation.

After the program, components of SPPB improved significantly in all subjects. The proportion of patients unable at admission to stand, walk and rise from a chair

Pulmonary Rehabilitation and COVID-19 
Table 2. Changes in $6 \mathrm{MWT}$ in 42 patients able to perform the test at admission and at the end of rehabilitation

\begin{tabular}{llll}
\hline & Before & After & $p$ value \\
\hline 6MWT, m & & & \\
$\quad$ Median (IQR) & $205.0(160.0-280.0)$ & $295.0(250.0-370.0)$ & 0.00 \\
Mean \pm SD & $229.0 \pm 102.5$ & $327.9 \pm 97.8$ & \\
6MWT, \% predicted & & & 0.00 \\
Median (IQR) & $46.0(32.0-55.0)$ & $70.0(56.7-75.2)$ & \\
Mean \pm SD & $47.7 \pm 18.9$ & $68.4 \pm 15.3$ & \\
\hline
\end{tabular}

Data are expressed as median (IQR) and mean \pm SD and range. $6 \mathrm{MWT}, 6-\mathrm{m}$ walking test; IQR, interquartile range. possible and effective in patients recovering from $\mathrm{CO}$ VID-19 infection, including those requiring assisted ventilation or oxygen therapy.

It has been reported that post-COVID patients can have an impaired physical functioning when they are discharged home, even after early mobilization [32]. Our results confirm those observations and extend to more severe patients directly transferred from acute care hospitals. As compared to that study [32], our patients suffered from more severe acute conditions as assessed by longer LoS in acute care hospitals and by the very high proportion of patients undergoing mechanical ventilation either invasive (including some tracheostomized) or NIV.

Among our patients, there were 16.1 and $4.4 \%$ with chronic obstructive pulmonary disease and asthma, respectively. The level and severity of comorbidities of our patients, as assessed by the CIRS indexes, were similar to those of patients reported outside the COVID conditions and likewise they did not influence the results of pulmonary rehabilitation [12].

Our pulmonary rehabilitation program was according to the Italian Position Paper [9]. Type, intensity, timing, and modality of intervention were tailored to the individual patient. Although the physical, cognitive, and emotional problems associated to prolonged LoS and/or mechanical ventilation are well-established in non-COVID-19 patients, their treatment is still under development [33-35].

Only patients with RT-PCR test for SARS-CoV-2 were included in our study. In the absence of such evaluation, there is no consensus on how long patients should be selfisolating. It has been suggested that local infection prevention recommendations should be followed with significant adaptation of the program, eventually with the adoption of "tele-rehabilitation" [36].

\section{Limitations of the Study}

For safety reasons, it was impossible to perform standard respiratory muscle or lung function tests, including the assessment of diffusion capacity. Hence, we are unable to define to what extent the decline in physical performance observed at admission can be ascribed to impairment in lung or respiratory muscle function. The results of an uncontrolled study may be difficult to interpret because we can suppose a positive effect in the long-term follow-up of these patients without a rehabilitative intervention. A control population not performing any activity would be unethical given the undisputed benefits of pulmonary rehabilitation or simple physical activity. One possible solution to this dilemma could be a trial with early versus delayed rehabilitation in post-COVID-19 patients. Our study could suffer from low external validity due to our restrictive inclusion criteria that limited the study to patients without functional limitations prior to COVID-19 so as to focus on the direct effect of the virus on muscle and functional ability, reducing confounding effects.

\section{Conclusion}

Pulmonary rehabilitation is possible and effective in patients recovering from COVID-19, including those requiring assisted ventilation or oxygen therapy. Our findings may be useful to guide clinicians taking care of patients surviving COVID-19.

\section{Statement of Ethics}

The research complies with the guidelines for human studies and was conducted ethically in accordance with the World Medical Association Declaration of Helsinki. 


\section{Conflict of Interest Statement}

The authors have no conflicts to declare.

\section{Funding Sources}

This work was partially supported by the "Ricerca Corrente" funding scheme of the Ministry of Health, Italy.

\section{Author Contributions}

The authors gave substantial contributions to the following: Zampogna E.: conception, interpretation, and drafting of the work; Paneroni M.: analysis, interpretation, and drafting of the work. Belli S.: conception and acquisition of the work; Aliani M.: conception and acquisition of the work; Gandolfo A.: conception and acquisition of the work; Visca D.: conception and acquisition of the work; Bellanti MT.: conception and acquisition of the work; Ambrosino N.: conception, interpretation, drafting of the work and revising it critically; Vitacca $\mathrm{M}$ : conception, interpretation, and revising it critically.

\section{References}

1 Covid-19 dashboard by the center for systems science and engineering (CSSE) at Johns Hopkins University. Available from: https:// gisanddata.maps.arcgis.com/apps/opsdashboard/index.html\#/bda $7594740 \mathrm{fd-}$ 40299423467b48e9ecf6. Accessed 2020 Oct 14.

$2 \mathrm{Wu} \mathrm{Z,} \mathrm{McGoogan} \mathrm{JM.} \mathrm{Characteristics} \mathrm{of} \mathrm{and}$ important lessons from the coronavirus disease 2019 (COVID-19) outbreak in China: summary of a report of 72,314 cases from the Chinese Center for disease control and prevention. JAMA. 2020;323(13):1239-42.

3 Huang C, Wang Y, Li X, Ren L, Zhao J, Hu Y, et al. Clinical features of patients infected with 2019 novel coronavirus in Wuhan, China. Lancet. 2020;395(10223):497-506.

4 Guan WJ, Ni ZY, Hu Y, Liang WH, Ou CQ, $\mathrm{He}$ JX, et al. Clinical characteristics of coronavirus disease 2019 in China. N Engl J Med. 2020;382(18):1708-20.

5 Vitacca M, Migliori GB, Spanevello A, Melazzini MG, Ambrosino N; COVID-19 ICS Maugeri IRCCS network, et al. Management and outcomes of post-acute COVID-19 patients in Northern Italy. Eur J Intern Med. 2020;78:159-60.

6 Paneroni M, Simonelli C, Saleri M, Bertacchini L, Venturelli M, Troosters T, et al. Muscle strength and physical performance in patients without previous disabilities recovering from COVID-19 pneumonia. Am J Phys Med Rehabil. 2021 Feb 1;100(2):105-109.

7 Vanhorebeek I, Latronico N, Van den Berghe G. ICU-acquired weakness. Intensive Care Med. 2020;46(4):637-53.

8 American Thoracic Society. Report of an adhoc international task force on early and short-term rehabilitative interventions in COVID-19 survivors. 2020 Apr. Available from: https://www.thoracic.org/members/ assemblies/assemblies/pr/journal-club/ report-of-an-ad-hoc-international-taskforce-to-develop-an-expert-based opinion. php?fbclid=IwAR1OKBl_clPGr5VdBQpmzkZ_JaTO5MPVSkNa9tYYFqooBRgNazimDPNW4ZA.
9 Vitacca M, Carone M, Clini EM, Paneroni M, Lazzeri M, Lanza A, et al. Joint statement on the role of respiratory rehabilitation in the COVID-19 crisis: the Italian position paper. Respiration. 2020;99(6):493-9.

10 Thomas P, Baldwin C, Bissett B, Boden I, Gosselink R, Granger CL, et al. Physiotherapy management for COVID-19 in the acute hospital setting: clinical practice recommendations. J Physiother. 2020;66(2):73-82.

11 Ceriana P, Nava S, Vitacca M, Carlucci A, Paneroni M, Schreiber A, et al. Noninvasive ventilation during weaning from prolonged mechanical ventilation. Pulmonology. 2019 Nov-Dec;25(6):328-33.

12 Maestri R, Bruschi C, Fracchia C, Pinna GD, Fanfulla F, Ambrosino N. Physiological and clinical characteristics of patients with COPD admitted to an inpatient pulmonary rehabilitation program: a real-life study. Pulmonology. 2019 Mar-Apr;25(2):71-8.

$13 \mathrm{NIH}$. Covid-19 treatment guidelines. Available from: https://www.covid19treatmentguidelines.nih.gov/therapeutic-options-under-investigation. Date last update: $2020 \mathrm{Apr}$ 21. Date Last access: 2020 May 12

14 Linn BS, Linn MW, Gurel L. Cumulative illness rating scale. J Am Geriatr Soc. 1968; 16(5):622-6.

15 Ippolito M, Vitale F, Accurso G, Iozzo P, Gregoretti C, Giarratano A, et al. Medical masks and respirators for the protection of healthcare workers from SARS-CoV-2 and other viruses. Pulmonology. 2020 Jul-Aug; 26(4):204-12.

16 Shah S, Vanclay F, Cooper B. Improving the sensitivity of the Barthel index for stroke rehabilitation. J Clin Epidemiol. 1989;42(8):703-9.

17 Bernabeu-Mora R, Medina-Mirapeix F, Llamazares-Herrán E, García-Guillamón G, Giménez-Giménez LM, Sánchez-Nieto JM. The short physical performance battery is a discriminative tool for identifying patients with $\mathrm{COPD}$ at risk of disability. Int $\mathrm{J}$ Chron $\mathrm{Ob}$ struct Pulmon Dis. 2015;10:2619-26.
18 Guralnik JM, Simonsick EM, Ferrucci L, Glynn RJ, Berkman LF, Blazer DG, et al. A short physical performance battery assessing lower extremity function: association with self-reported disability and prediction of mortality and nursing home admission. J Gerontol. 1994 Mar;49(2):M85-94.

19 Bergland A, Strand B. Norwegian reference values for the short physical performance battery (SPPB): the Tromsø Study. BMC Geriatr. 2019;19(1):216.

20 Perera S, Mody SH, Woodman RC, Studenski SA. Meaningful change and responsiveness in common physical performance measures in older adults. J Am Geriatr Soc. 2006;54(5): 743-9.

21 Studenski S, Perera S, Patel K, Rosano C, Faulkner K, Inzitari M, et al. Gait speed and survival in older adults. JAMA. 2011;305(1): 50-8.

22 Guralnik JM, Ferrucci L, Simonsick EM, Salive ME, Wallace RB. Lower-extremity function in persons over the age of 70 years as a predictor of subsequent disability. $\mathrm{N}$ Engl J Med. 1995;332(9):556-61.

23 Holland AE, Spruit MA, Troosters T, Puhan MA, Pepin V, Saey D, et al. An official European Respiratory Society/American Thoracic Society technical standard: field walking tests in chronic respiratory disease. Eur Respir J. 2014;44(6):1428-46.

24 Enright PL, Sherrill DL. Reference equations for the six-minute walk in healthy adults. Am J Respir Crit Care Med. 1998;158(5 Pt 1): 1384-7.

25 Borg G. Psychophysical basis of perceived exertion. Med Sci Sports Exerc. 1982;14:377-81.

26 Maltais F, LeBlanc P, Jobin J, Bérubé C, Bruneau J, Carrier L, et al. Intensity of training and physiologic adaptation in patients with chronic obstructive pulmonary disease. Am J Respir Crit Care Med. 1997;155(2):555-61.

27 Winck JC, Ambrosino N. COVID-19 pandemic and non invasive respiratory management: every Goliath needs a David. An evidence based evaluation of problems. Pulmonology. 2020 Jul-Aug;26(4):213-20. 
28 Polastri M, Nava S, Clini E, Vitacca M, Gosselink R. COVID-19 and pulmonary rehabilitation: preparing for phase three. Eur Respir J. 2020;55(6):2001822.

29 George PM, Barratt SL, Condliffe R, Desai SR, Devaraj A, Forrest I, et al. Respiratory followup of patients with COVID-19 pneumonia. Thorax. 2020 Aug 24. Available from: 10.1136/thoraxjnl-2020-215314. Epub ahead of print.

30 Spruit MA, Holland AE, Singh SJ, Tonia T, Wilson KC, Troosters T. COVID-19: interim guidance on rehabilitation in the hospital and post-hospital phase from a European Respiratory Society and American Thoracic Societycoordinated international task force. Eur Respir J. 2020 Aug 13;56(6):2002197. Online ahead of print.
31 Available from: https://iris.paho.org/bitstream/handle/10665.2/52035/NMHMHCOVID19200010_eng.pdf? sequence $=6 \&$ isAllowed $=\mathrm{y}$. Accessed 2020 Oct 16 .

32 Belli S, Balbi B, Prince I, Cattaneo D, Masocco F, Zaccaria S, et al. Low physical functioning and impaired performance of activities of daily life in COVID-19 patients who survived hospitalisation. Eur Respir J. 2020;56(4): 2002096.

33 Brown SM, Bose S, Banner-Goodspeed V, Beesley SJ, Dinglas VD, Hopkins RO, et al. Approaches to addressing post-intensive care syndrome among intensive care unit survivors. A narrative review. Ann Am Thorac Soc. 2019;16(8):947-56.
34 Kiekens C. Follow-up services for improving long-term outcomes in intensive care unit (ICU) survivors: a cochrane review summary with commentary. J Rehabil Med. 2019;51: 879-82.

35 Puthucheary ZA, Denehy L. Exercise intervention in critical illness survivors: understanding inclusion and stratification criteria. Am J Respir Crit Care Med. 2015;191(12): 1464-7.

36 Mukaino M, Tatemoto T, Kumazawa N, Tanabe S, Katoh M, Saitoh E, et al. Staying active in isolation: telerehabilitation for individuals with the severe acute respiratory syndrome coronavirus 2 infection. Am J Phys Med Rehabil. 2020;99(6):478-9. 